Livraisons

d'Histoire

de l'Architecture

\section{Livraisons de l'histoire de l'architecture}

28 | 2014

Jean-Louis Pascal

\title{
Avant la bibliothèque
}

Before the National Library

Vor der Bibliothèque Nationale

\section{Anne Richard-Bazire}

\section{CpenEdition}

\section{Journals}

Édition électronique

URL : http://journals.openedition.org/lha/392

DOI : 10.4000/lha.392

ISSN : 1960-5994

Éditeur

Association Livraisons d'histoire de l'architecture - LHA

Édition imprimée

Date de publication : 10 décembre 2014

Pagination : 49-70

ISSN : 1627-4970

Référence électronique

Anne Richard-Bazire, «Avant la bibliothèque », Livraisons de l'histoire de l'architecture [En ligne], 28 | 2014, mis en ligne le 10 décembre 2016, consulté le 19 avril 2019. URL : http://

journals.openedition.org/lha/392 ; DOI : 10.4000//ha.392

Ce document a été généré automatiquement le 19 avril 2019

Tous droits réservés à l'Association LHA 


\section{Avant la bibliothèque}

Before the National Library

Vor der Bibliothèque Nationale

Anne Richard-Bazire

1 Pascal réalise ses deux premières œuvres assez vite après l'obtention de son grand prix : la maison-atelier que son maître et ami William Bouguereau lui demande d'élever rue Notre-Dame-des-Champs à Paris et une demeure à la campagne aux environs de Bayeux, le château d'Escures près de Commes, dans le Calvados (ill. 1), qu'il construit en profitant sans doute des quelques passages qu'il fait à Paris vers la fin de sa pension romaine. 


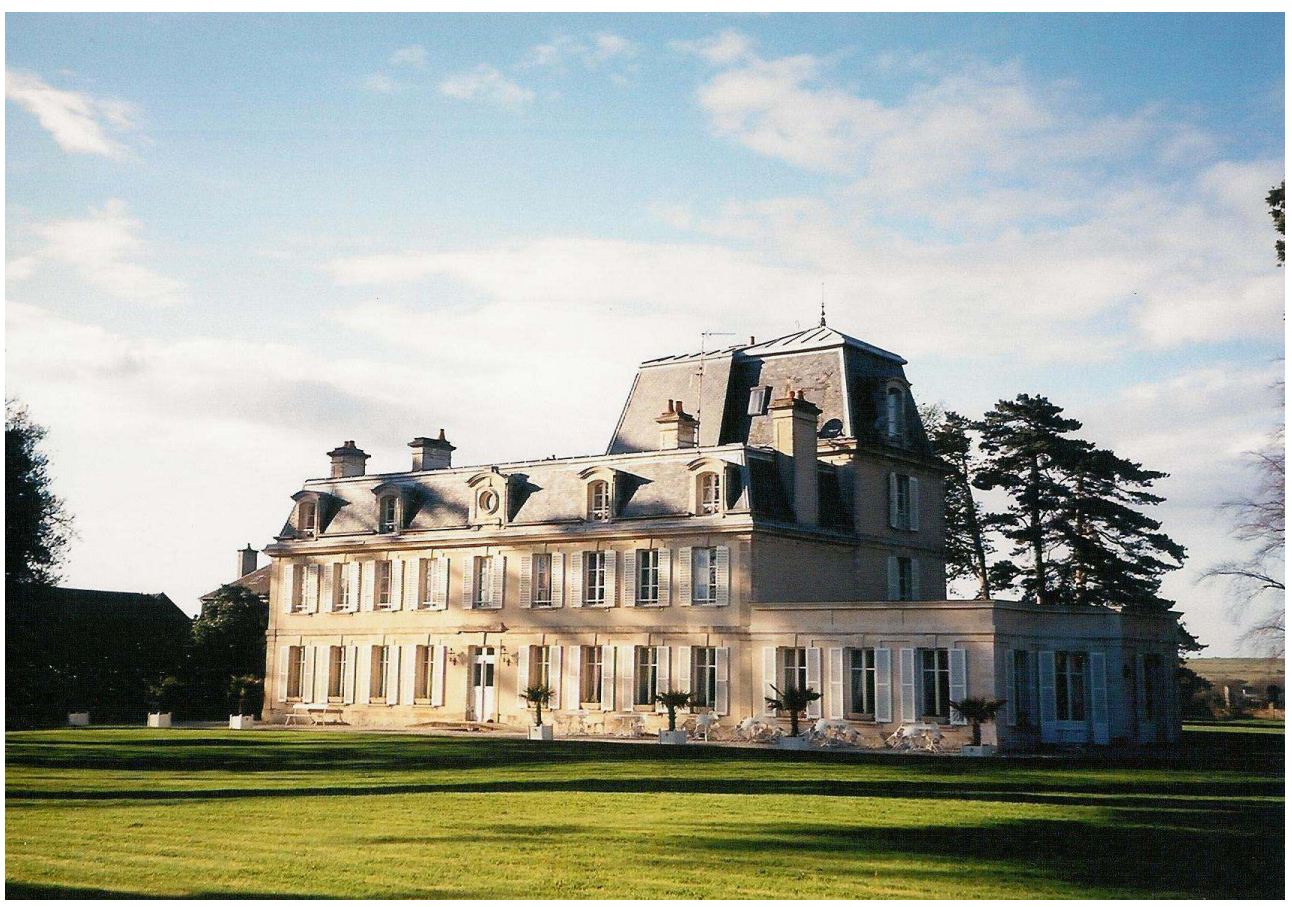

1869. Façades sud et est

Cl. Auteur

2 Il obtient aussi la commande d'un monument dédié au colonel d'Argy dans l'église SaintLouis-des-Français de Rome. Ce sont toutes trois des œuvres de jeunesse qui montrent la sobriété du classicisme du jeune architecte. Les années soixante-dix sont aussi celles de deux échecs : Jean-Louis Pascal participe à deux des grands concours publics du XIX siècle : le concours pour la reconstruction de l'Hôtel de Ville de Paris ${ }^{1}$ (1873) et celui pour la construction de la basilique du Vœu national au Sacré-Cœur (1874). La décoration des chapelles de la cathédrale Saint-Louis de la Rochelle (ill. 2) lui donne l'occasion, à partir de 1873 de réaliser pour la première fois des décors sacrés. 


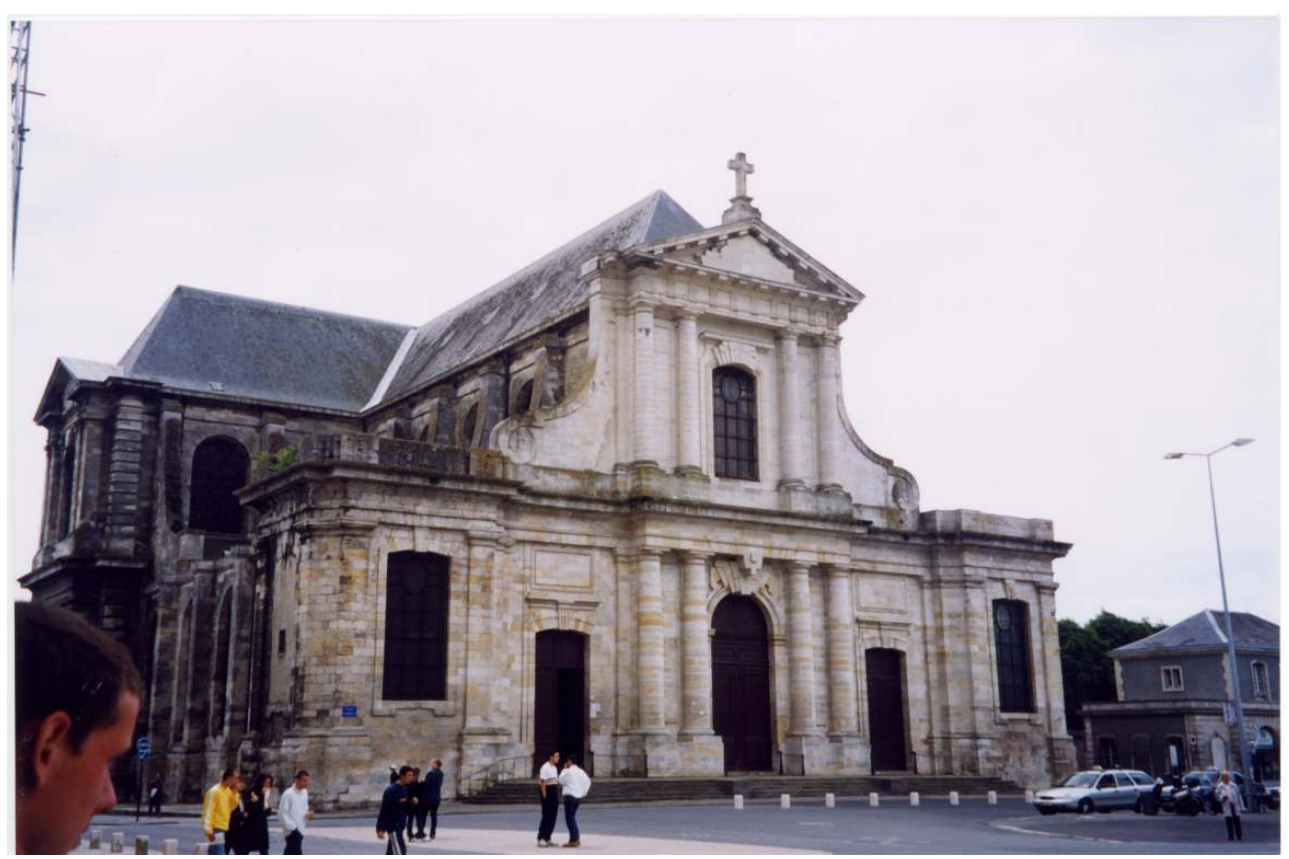

Façade. Architectes : Jacques V Gabriel (plans), Jacques Ange Gabriel (1742), Antoine Brossart (1849-1862)

$\mathrm{Cl}$. auteur

\section{Les débuts d'un jeune architecte}

\section{L'HÔTEL PARTICULIER DE WILLIAM BOUGUEREAU (1868)}

3 En septembre 1868, William Bouguereau emménage avec sa famille et sa mère dans l'hôtel particulier récemment achevé au ${ }^{\circ} 75$ de la rue Notre-Dame-des-Champs (Paris $6^{\mathrm{e}}$ ), qu'il a construit grâce à ses premières économies. Cette nouvelle résidence est située dans un quartier tranquille :

"Avant la Révolution, la rue Notre-Dame-des-Champs était un chemin peuplé de monastères. [...] Aujourd'hui les prieurés ont en partie disparu, mais c'est toujours un Paris spécial, plongé dans le calme et le recueillement. Seulement, au lieu des murailles blafardes des cloitres, de hautes et tristes maisons bordent la rue de chaque côté. Des ateliers comme des rideaux de théâtre, se découpent dans chaque façade. [...] Notre cher Baudry est mort au $n^{\circ} 70$ et Rosa Bonheur ${ }^{2}$, le grand animalier, occupait le $n^{\circ} 61$ [...].Cette rue, si on la débaptise un jour, devrait à juste titre s'appeler la voie Appienne de la peinture $»^{3}$.

Cette rue desservait aussi les ateliers des peintres Jean-Paul Laurens (1838-1921) ${ }^{4}$, Alfred de Curzon (1820-1895) ${ }^{5}$ et Émile Auguste Carolus-Duran ${ }^{6}$ (1838-1917).

5 En 1866, Jean-Louis Pascal vient de remporter le grand prix de Rome, lorsque le peintre le choisit pour édifier sa maison. Ami de William Bouguereau, Paul Baudry, qui commence à travailler en 1864 à la réalisation des peintures du grand foyer de l'Opéra de Paris alors que Jean-Louis Pascal fait partie de l'agence de Charles Garnier en compagnie du jeune frère de Paul, Ambroise, a peut-être renforcé Bouguereau dans cette décision de choisir ce jeune architecte et ancien élève pour lui édifier son atelier et sa maison. 
6 Un autre ami de William Bouguereau, le peintre Jules Joseph Lefebvre, a fréquenté JeanLouis Pascal à l'École des beaux-arts puis à la Villa Médicis. William Bouguereau était un ami de Rodolphe Julian (1839-1907) et c'est avec beaucoup d'intérêt qu'il avait suivi la fondation, par celui-ci, du premier atelier libre à Paris, en 1868, au 27 passage des Panoramas. Si Jules Lefebvre est l'un des tous premiers professeurs de l'Académie Julian, ce n'est qu'en 1875 que William Bouguereau accepte la direction d'un atelier dans cette école $^{7}$. Il y fera d'ailleurs la connaissance de sa seconde épouse ${ }^{8}$ : Elizabeth Jane Gardner, élève de Jules Lefebvre, l'Académie Julian étant la première école parisienne à accepter les femmes.

Jean-Louis Pascal réalise pour William Bouguereau une maison relativement sobre :

«Elle tient, comme apparence extérieure, à la fois de la maison de rapport et de l'hôtel privé : l'un d'ailleurs est contigu à l'autre, sous le même toit, avec façades sur rue, sur une venelle de cité d'artistes, et sur des jardins. Sauf l'atelier, occupant le dernier étage et invisible du dehors, rien ne distingue cette habitation de la demeure confortable d'un industriel, d'un homme de robe, ou d'un fonctionnaire de haut rang ".

8 Côté rue, la façade, traitée avec sobriété, est une façade haussmannienne typique des années 1850 à $1870^{10}$ : rez-de-chaussée et entresol sont striés de refends horizontaux; la transition avec le premier étage est assurée par un balcon de fer forgé reposant sur de robustes consoles, qui marque la salle de réception du bel étage. Les fenêtres à encadrement de celle-ci sont mises en valeur par des frontons triangulaires supportés par de petites consoles, tandis que les autres fenêtres du premier étage sont ornées de simples dais, également sur consoles. Fenêtres du rez-de-chaussée et de l'entresol d'une même travée sont unies par un encadrement mouluré; seule rupture dans cette verticalité : l'allège des fenêtres de l'entresol ornée d'une table rectangulaire avec disque central. L'architecte a tiré un bandeau profilé entre l'entresol et les autres étages, de manière à limiter la dimension des pilastres engagés colossaux encadrant les fenêtres de ceux-ci. Au-dessus de la corniche, dont le profil reprend celui du bandeau de séparation, sont percées dans le brisis du toit à la Mansart, de petites lucarnes à frontons triangulaires. Agrémentées de persiennes, elles ajoutent un troisième étage à cet hôtel particulier.

Une grille donne accès à la ruelle privée longeant le côté gauche de l'habitation. La porte d'entrée s'ouvre tout de suite sur la droite : dans un petit hall dont le mur de gauche est agrémenté d'une fontaine, à l'eau ruisselant dans une coquille, s'élève un escalier tendu à l'époque de tentures sombres ; il mène à une antichambre sans gravure et à un salon riche et sévère, dont le plafond est décoré d'une peinture en caisson central représentant les Heures du Jour.

«Aux murs, des portraits de famille, quelques tableaux anciens et modernes; un mobilier fort simple; et çà et là, sur les consoles et sur les tables, des jardinières, des albums et quelques bibelots féminins $»^{11}$.

10 À l'arrière de la maison s'étend un joli jardin agrémenté de sculptures à l'antique. De grands arbres et des arbustes de haute taille cachent les murs décorés de pots.

«Pour conserver à son atelier une belle lumière, et à son jardin la physionomie rustique qu'il avait au temps, peu lointain, où le terrain était tout en terrains d'agrément et de culture, l'artiste a acheté les terrains environnants, et n'y a laissé élever que des constructions légères, très basses, des ateliers de sculpteurs, garnis, eux aussi, de plantes grimpantes, et entourés de plantes et de fleurs $»^{12}$. 
11 C'est sur ce jardin que donne l'atelier de William Bouguereau, dont la grande baie vitrée, à l'arc surbaissé, est surmontée d'une tête de Minerve, déesse des arts, au casque ailé surmonté de son attribut, la chouette (ill. 3).

\section{4 : Château d'Escures à Commes, Calvados}

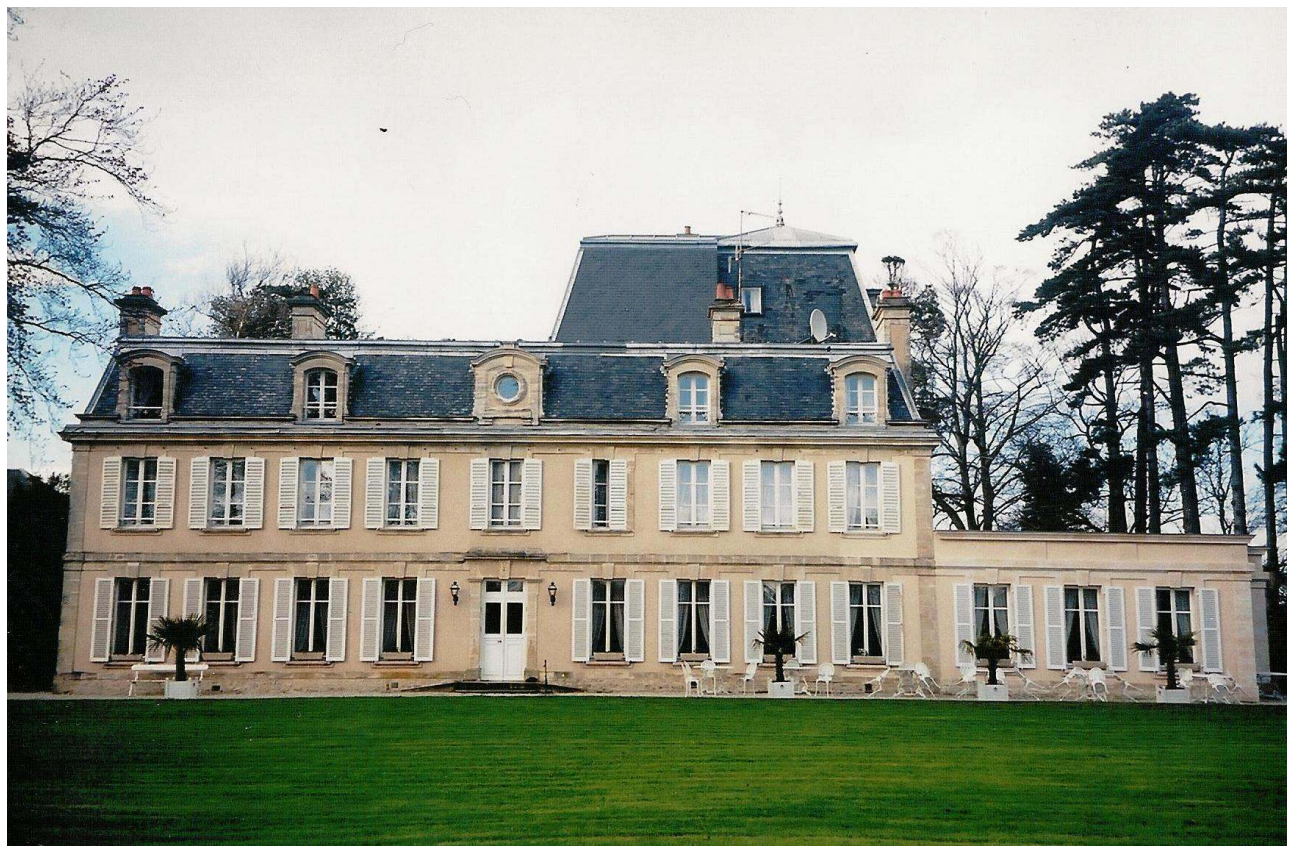

Architecte Jean-Louis Pascal. À partir de 1869. Logis, façade principale au sud

$\mathrm{Cl}$. auteur

Cette baie ressemble si étrangement à la grande arcade sommée d'une tête de Minerve qui éclaire la façade postérieure de l'Opéra de Paris ${ }^{13}$, que l'on peut se demander si l'on ne s'en est pas inspiré en en proposant ici une version simplifiée.

13 L'atelier occupe toute la moitié nord du dernier étage ; il mesure : 11,50 mètres sur 9,50 mètres et s'élève à une hauteur de 6,9 mètres. Le mur qui donnait au nord, entièrement vitré du plancher au plafond, était garni d'une lourde draperie destinée à faire varier la lumière. Celle-ci pénétrait aussi par un châssis vitré de 4 mètres sur 2,50 mètres, situé au centre du plafond, dont la partie inférieure, à fleur avec le plafond, était en verre armé diffusant ; un rouleau de tissus opaque, coulissant par un jeu de poulies sur deux rails, permettait de régler l'intensité de la lumière. À l'origine, la grande fenêtre centrale était agrémentée d'un vaste balcon en saillie, dont toutes les faces étaient vitrées, pour ne pas gêner la pénétration de la lumière. Cette partie vitrée de l'atelier, qui ressemblait à une serre, fut démontée bien après la mort de William Bouguereau. Aujourd'hui ne subsiste qu'un étroit balcon avec une grille ${ }^{14}$. Paul Eudel, nous donne, en 1886, une description très précise de l'atelier du peintre ${ }^{15}$ :

"Son atelier, suivant l'usage, est dans les combles, "à l'entresol des pierrots" comme disent les rapins. Montons sur un tapis de sparterie l'escalier magnifique qui conduit à la vaste et haute salle où se trouvent ses chevalets. Tout d'abord, une véritable déception. Aucun bibelot, aucune prétention à l'élégance. Pas d'armures ni de meubles gothiques. Ce n'est nullement un magasin de bric-à-brac comme dans certains ateliers somptueux où l'on peut $y$ faire tout excepté de la peinture. Ici, au contraire, on doit bûcher ferme. Les yeux n'ont rien pour les distraire. Sans s'égarer, ils peuvent rester fixés sur le modèle ou sur la toile [...] Une cloison de deux mètres de haut environ, recouverte de vieilles tapisseries assez médiocres, 
coupe l'atelier dans toute sa longueur. Contre cette séparation est adossé un beau régulateur en ébène, de l'époque Louis XIV, aux cuivres finement ciselés, aux hanches arrondies dans une courbe fine et gracieuse [...] Au bas des murs peints en terre cuite s'accotent des cartons bondés de dessins et de croquis [...] Dans la section de l'atelier dissimulée par la cloison dont nous avons parlé plus haut, [...] la vie semble moins dispersée, moins éparpillée. Çà et là, des chaises modernes, une armoire normande, un divan vert foncé et un fauteuil en soie blanche brochée de rouge, poussé près d'une table couverte de papiers, d'enveloppes, de carnets et de cartes de visite $[. .$.$] »$

Ce type d'immeuble, appelé aujourd'hui haussmannien, apparait alors, présentant une façade régularisée accentuée par un bandeau horizontal entre les étages, raccordé à l'immeuble voisin et aligné sur la rue. Et lorsque, plus tard, la typologie de l'immeuble à cinq étages se généralisera dans Paris, certains secteurs continueront d'affirmer leur vocation résidentielle. C'est le cas des abords du jardin du Luxembourg :

"Ici, le modèle à quatre étages s'est maintenu, offrant une élévation identique de soubassement et de retiré, mais avec deux étages principaux au lieu de trois. Ce système correspond mieux [...] à la tradition académique, car il permet l'intégration de l'ordre colossal [...] L'ambiance urbaine n'est pas du tout la même: des immeubles plus trapus, des façades au contact de la rue [...], une animation plus discrète ${ }^{16}$.

Plus tard, Pascal réalisera quelques modifications à l'hôtel : «J'ai pris rendez-vous avec la fille de Bouguereau, Madame Vincens, pour diverses transformations à l'hôtel de son père » écrit-il au sculpteur Gustave Germain (1848-1909), alors qu'il prend les eaux à Bourbon-Lancy en août $1905^{17}$.

\section{LE CHÂTEAU D'ESCURES À COMMES, CALVADOS (1869) ${ }^{18}$}

Ce sobre classicisme, nous le retrouvons dans le petit château d'Escures, que Pascal construit à partir de 1869 pour le sieur Gosset, à Commes, dans le Calvados. D'un château initial, figurant sur le cadastre de 1809 , ne subsiste qu'un corps secondaire bordant à l'ouest la cour des communs qui comprenait une forge et une scierie, une étable, des écuries, une sellerie et deux remises avec logement et resserre à fruits à l'étage. Le corps principal du logis, en moellon enduit, à un étage carré et un étage de comble (ill. 4), présente une façade ordonnancée: neuf fenêtres au premier étage, huit au rez-dechaussée de part et d'autre de la porte d'entrée qu'encadrent des pilastres engagés toscans. Des pierres de taille en chaînage renforcent les angles du bâtiment, et séparent à l'aide d'un bandeau le rez-de-chaussée de l'étage ; un linteau de pierre surmonte aussi les baies avec claveau proéminant à la clef. Cinq lucarnes agrémentent le toit brisé recouvert d'ardoises ; celle du milieu présente un oculus. À l'arrière du bâtiment, au nord-est, un pavillon carré à deux étages est surmonté d'un toit brisé en pavillon. En 1873, Pascal édifie le long de la façade nord une galerie avec rez-de-chaussée en pierre de taille, étage en bois et toit en appentis. Il ajoute à la façade est une véranda (ill. 5) détruite dans le courant du XXe siècle et remplacée par la suite par un pavillon bas. 


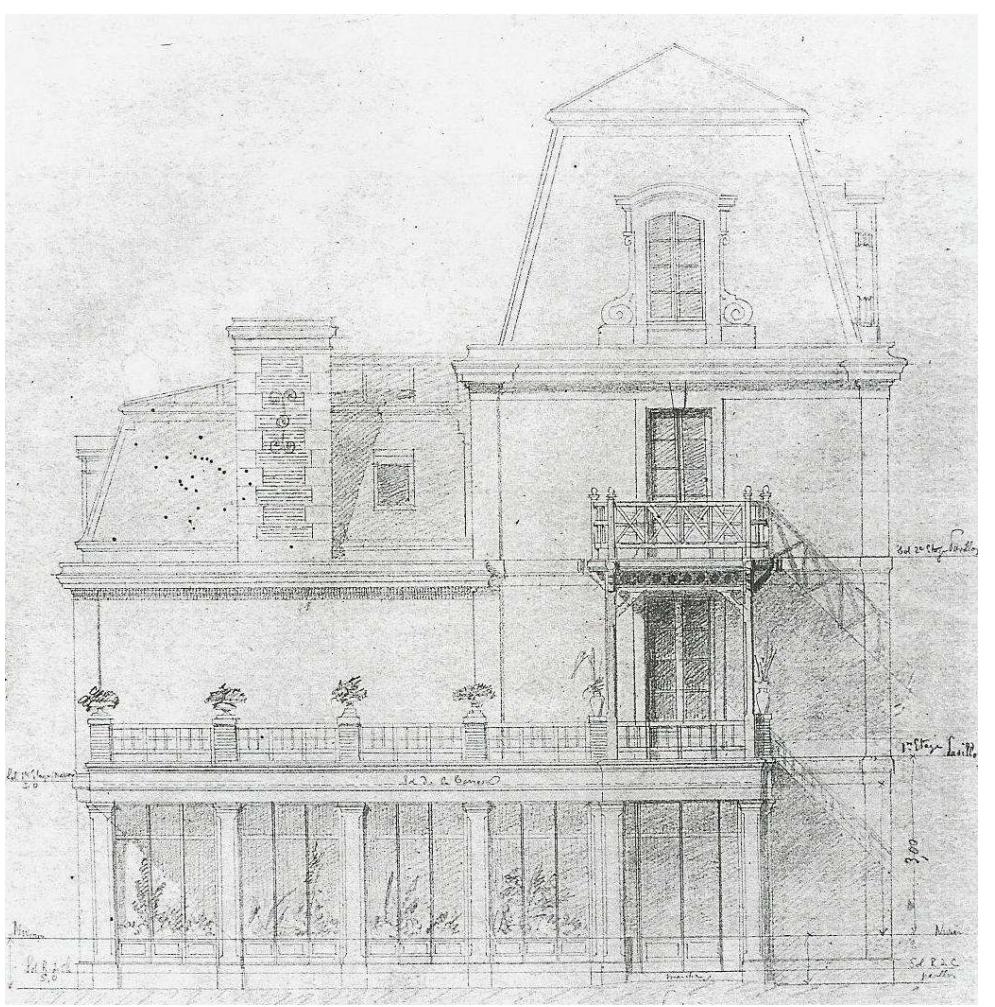

Architecte Jean-Louis Pascal. 1873. Façade est, $7^{\text {re }}$ variante du projet de véranda. Monsieur Gosset à Commes, ensemble de 9 dessins à l'encre sur calque de la galerie nord et de la véranda est par JeanLouis Pascal, coll. privée

Service régional de l'Inventaire de Basse-Normandie, dossier établi par Bernard Ducouret, doc. 7 , photo Inv. Ph. Corbierre, 91.14.2270.XB, 2520.XBA, 2519.XBA

17 À l'avant de la propriété s'étend un joli jardin paysager avec un ahah au nord ; à l'arrière, un jardin potager (derrière les communs) et une serre en verre avec toit en appentis à l'extrémité nord-est de la propriété. En 1909, l'architecte de Bayeux, E. Lair, transforme l'aménagement du $2^{\mathrm{e}}$ étage ; probablement à la même époque, on construit de nouvelles écuries en bordure du jardin potager et on installe une scie à ruban. La galerie nord sera fermée et augmentée d'un toit brisé avec bow-window sur deux niveaux lors de l'aménagement en hôtel de voyageurs : l'hôtel de la Chenevière au XX $\mathrm{X}^{\mathrm{e}}$ siècle.

\section{Quelques monuments commémoratifs}

\section{Le monument au colonel d'Argy (1870)}

Au début de l'année 1870, on confie à Jean-Louis Pascal la réalisation d'un monument commémoratif dans l'église Saint-Louis-des-Français de Rome ${ }^{19}$ en l'honneur du comte Henri Charles Louis d'Argy, premier colonel de la Légion romaine, mort à Rome le 26 janvier 1870. Il dispose de huit à neuf cents francs, sans honoraires : «Je suis tout fier de laisser à Rome cette trace de mon passage, mais le diable est de rentrer dans le chiffre $! »^{20}$ . Le monument est apposé contre l'un des piliers qui sépare la nef du bas-côté gauche de l'église ; une large plaque rectangulaire de marbre coloré et veiné, encadrée de marbre blanc, contient le texte commémoratif ${ }^{21}$; dans la partie haute, un fronton semi-circulaire 
interrompu surmonte un large médaillon et un buste de bronze porté par une console blanche. Le sculpteur Charles Jean Marie Degeorge a prêté gracieusement son concours à Jean-Louis Pascal. C'est aussi lui qui aide Pascal à réaliser le beau monument à Henri Regnault dans la cour du mûrier de l'École des beaux-arts à Paris.

\section{Le monument à Henri Regnault (1872-1876)}

« Nous voici maintenant dans la cour du mûrier ${ }^{22}$. Décorée en style néo-grec, ornée d'antiques sculptés par d'anciens pensionnaires, le vieux cloître des Petits Augustins, patio paisible au murmure de sa vasque, invite d'autant plus au recueillement que, sous les arcades du portique, on rencontre, dans un angle, le monument de Regnault $»^{23}$.

En 1866, l'architecte Charles Chauvin (1820-?) avait, sous la direction de son maître Duban, transformé la cour du mûrier de l'École des beaux-arts en un véritable atrium. Il avait aussi décoré les galeries de l'École d'après les Loges de Raphaël et collaboré à la maison pompéienne de Jérôme Napoléon ${ }^{24}$. Jean-Louis Pascal réalise dans la cour du mûrier, en collaboration avec le sculpteur Charles Jean Marie Degeorge et l'architecte Ernest Georges Coquart, le monument à Regnault et aux artistes morts pour la France pendant la guerre de 1870 (ill. 6).

\section{6 : Monument à Henri Regnault et aux élèves de l'E.B.A.}

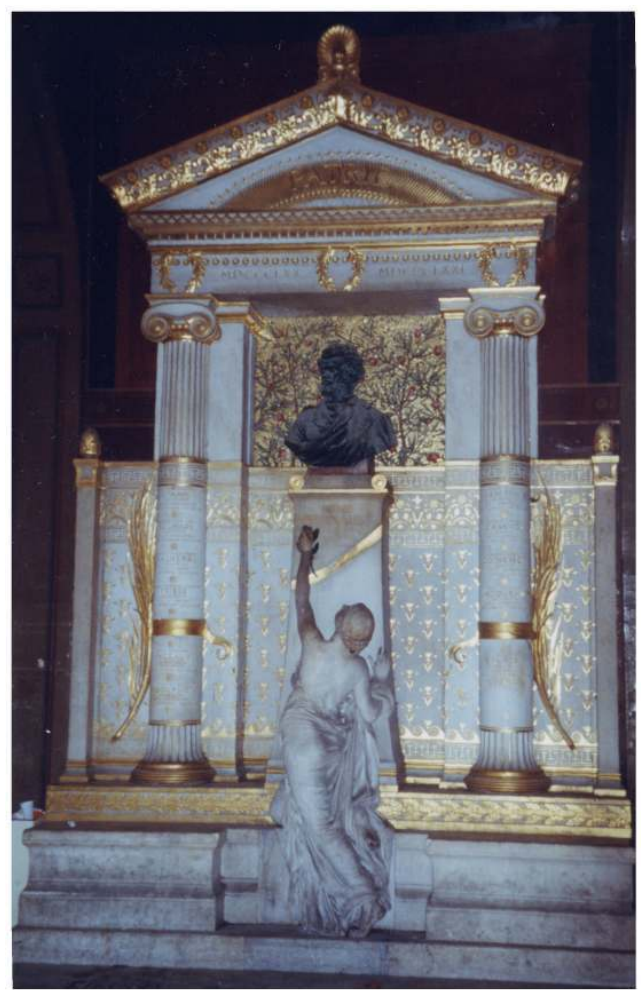

Jean-Louis Pascal et Ernest Coquart, architectes. 1872-1876. Marbre blanc, décorations polychromes et or. École nationale supérieure des beaux-arts (cour du mûrier). Sculpteurs : Henri Chapu : La Jeunesse, médaillée d'or au Salon de 1875, Charles Marie Degeorge : buste en bronze de Regnault exposé au Salon de 1876. Sculpture d'ornement: Perrin. Mosaïque : Jean-Dominique Facchina. Dorure et peinture : Charles Chauvin. Branche de laurier : Maison Barbedienne

$\mathrm{Cl}$. auteur 
20 Coquart en tant qu'architecte des bâtiments civils, était chargé de l'achèvement de l'École des beaux-arts ${ }^{25}$ (il restaure la cour du mûrier, décore le grand hall du musée des Études et y dispose la collection des divers moulages). la médaille d'honneur au Salon de 1875.

«Il ne s'agissait plus d'un monument érigé à l'air libre, mais d'une sorte de grande stèle placée dans le demi-jour d'un cloître. Aussi le détail est-il beaucoup plus fin. Certains ornements ont la précision des tableaux du quattrocento, œuvres de marbriers qui furent aussi des ciseleurs, et la polychromie du fond, la mosaïque, les rehauts d'or sur lesquels se détache la statue de Chapu, donnent à cet ensemble son charme et sa préciosité ${ }^{26}$.

C'était un usage en honneur entre pensionnaires de l'Académie de France à Rome : Pascal et Degeorge qui avaient eu le grand prix la même année que Regnault furent désignés pour lui élever ce cénotaphe dans un angle de l'ancien cloitre. Jean-Louis Pascal avait beaucoup admiré Henri Regnault lors de son séjour à la Villa Médicis et il avait mis toute son âme dans cette composition :

"Cette heureuse évocation de l'Antiquité grecque en l'honneur de nos héros nous enthousiasmait, notre bruyante jeunesse devenait rêveuse en passant dans cette galerie et nous comprenions mieux les maximes de notre cher patron: "Puisez toujours vos inspirations aux sources de l'art" ${ }^{27}$.

Le monument de marbre blanc, figurant une sorte de petit temple, est adossé à un mur dans lequel sa partie médiane est même légèrement engagée ; il est sobrement décoré d'ornementations polychromes ${ }^{28}$. Il se compose de deux colonnes d'ordre ionique soutenant un fronton triangulaire. Sur le seuil du petit temple, entouré d'une enceinte dessinée en bas-relief, deux bancs semblent inviter le passant à se recueillir en songeant aux héros morts pour la patrie. Une draperie figurée, semée de lotus d'or, fleur de l'immortalité, masque l'entrée du temple. Entre les deux colonnes, un cippe funéraire, sur lequel est inscrit : « Henri Regnault tué à Buzenval le 19 janvier 1871 », sert de piédestal au buste en bronze de Regnault dû au peintre et sculpteur d'ornement Charles Jean Marie Degeorge, buste qui fut exposé au Salon de 1876 et dont l'énergique visage marque une noble et suprême indignation. Coquart, lui, voulait un buste droit et sans idée, plutôt qu'un buste à effet, pensant que " la tête si expressive de Regnault suffi[sai]t parfaitement à exprimer tout le drame de la composition ». Le monument sert d'écrin à la Jeunesse ${ }^{29}$ d'Henri Chapu (1833-1891), jeune femme appuyée d'un genou sur le cippe portant le buste d'Henri Regnault et dont les formes juvéniles sont tendues dans un geste élégant pour offrir au jeune héros la palme du martyr, le rameau de la gloire. À cette fin, Jean-Louis Pascal demande à Charles Garnier de lui envoyer, s'il en a, des feuilles du laurier victorieux qui dessine les longues allées de la Villa Médicis, car il a besoin d'un rameau à mettre dans la main de la figure de la Jeunesse. Celui-ci explique qu'il ne trouve à Paris que le laurier amande et le laurier sauce dont les feuilles, fermes et jolies, n'ont pas le sens symbolique qu'il leur désire ${ }^{30}$. L'attitude du jeune héros est énergique et fière; il porte le costume militaire ; la tête, tournée, est comme menaçante. Charles Degeorge a bien rendu les qualités hérö̈ques (courage, ardeur, violence même), malgré la difficulté d'obtenir une ressemblance de souvenir et sans la nature. Derrière ce buste, un fond de mosaïque où se détachent sur un ciel d'or les lauriers d'un jardin victorieux ${ }^{31}$.

Henri Chapu, élève de James Pradier (1792-1862) à l'École des beaux-arts, grand prix de Rome en 1855 , avait poursuivi une carrière très officielle, travaillant notamment pour l'Opéra, l'église Saint-Étienne-du-Mont, l'Hôtel de Ville de Paris et la Sorbonne. La 
Jeunesse est le meilleur exemple de ses figures drapées aux regards inspirés, aux gestes touchants, grâce auxquels il compose des jeux de lumière discrets et harmonieux.

$\mathrm{Au}$ fronton du petit temple resplendit, comme dans les rayons d'un couchant ou d'une aurore, le mot «PATRIE " $^{32}$, résumé de tout le monument, d'où s'échappe dans l'antéfixe du couronnement, la flamme, symbole de résurrection et d'espérance. Dans la cimaise du fronton rampent les feuilles de chêne et les fleurs de pavot du sommeil éternel. Entre les trois couronnes de feuilles de laurier de l'architrave s'insèrent les deux dates : 1870-1871. La guirlande de feuilles de chêne et de glands ornant la bande du soubassement symbolise le courage civique ${ }^{33}$. Aux angles du fronton sont perchées deux chouettes, oiseaux lugubres et sinistres allusions à la laideur de la mort et à la tristesse du souvenir (peutêtre aussi à l'oiseau de Minerve, déesse des artisans). Les colonnes sont cannelées et dorées dans leurs parties haute et basse. Sur la partie intermédiaire, lisse, sont inscrits, en lettre d'or, le nom des autres artistes morts pendant la guerre.

Sur le côté du piédestal du buste sont sculptés la palette de peintre, les brosses, l'appuimain et une branche d'olivier, symbole des succès de l'artiste ${ }^{34}$.

L'initiative de la fondation du monument était due à Jean-Baptiste Eugène Guillaume (1822-1905), directeur de l'École des beaux-arts qui, dès le 28 janvier 1871, proposait à Jules Simon, ministre de l'Instruction publique, des cultes et des beaux-arts d'élever dans le cloître de l'école cet édicule destiné à perpétuer la mémoire du jeune héros mort au champ d'honneur ${ }^{35}$. Les frais d'exécution furent couverts par une subvention du ministère de l'instruction publique et des beaux-arts ${ }^{36}$ et par une souscription privée ${ }^{37}$ dont le produit total atteignit quinze mille cinq cents francs ${ }^{38}$. Tous les collaborateurs de l'œuvre avaient d'ailleurs tenu à ne tirer aucun bénéfice de leur travail. L'État avait fourni les marbres. Un avant-projet fut présenté à l'approbation du ministre et contresigné par lui, le 28 novembre 1872. Les artistes furent secondés par Perrin pour la sculpture d'ornement, sur des dessins donnés en grandeur d'exécution par les architectes, JeanDominique Facchina (1826-1903) pour la mosaïque, et Charles Chauvin, peintre décorateur de l'École, pour la dorure et les fonds décorés. La marbrerie fut confiée à la maison Drouet et Lozier. Barbedienne exécuta la branche de laurier de la figure de la Jeunesse. Le monument avait été commandé en 1872. En 1874, Jean-Louis Pascal écrit à Louise Garnier : « Toujours est-il que Chapu a terminé l'esquisse de notre figure, que nous avons fait choix de nos morceaux de marbre et que l'exécution va commencer [...] La figure de Chapu ne sera pas finie avant un an puisqu'il s'agit d'un marbre. Il ne faut guère donc espérer l'inauguration avant le 19 janvier $1875 »^{39}$. Des retards vont intervenir car Chapu, n'ayant rien d'autre à présenter au Salon, veut y exposer son marbre : « Nous ne pouvons pas poser la troisième pierre sans avoir sa stèle et sa figure. On ne pourra commencer à bâtir avant le mois de juin ", écrit Jean-Louis Pascal en février $1875^{40}$. L'inauguration se déroula le 12 août 1876, après la distribution des récompenses décernées aux artistes qui avaient figuré comme exposants au Salon de 1876 et des récompenses accordées aux élèves de l'École pour leurs travaux de l'année. La courte cérémonie fut présidée par Charles Philippe de Chennevières (1820-1899), directeur des Beaux-Arts ${ }^{41}$. Deux autres monuments furent élevés à Henri Regnault : l'un dans le parc de Buzenval où il avait été tué, l'autre à l'intérieur du lycée Henri IV où il avait fait ses études. Son corps fut inhumé dans la sépulture de sa famille, au cimetière sud ${ }^{42}$.

Jean-Louis Pascal avait-il eu une prémonition lorsqu'il écrivait de Florence, en 1868, à Charles Garnier : 
«Peut-être avez-vous vu l'aventure qui a failli me priver de mon peintre et peut-être notre temps d'un artiste de talent. Regnault a fait une chute de cheval si effroyable qu'il a failli rester sur le coup. Tout travail lui a été longtemps interdit [...] Il est enfin en bonne voie de guérison mais je ne le crois pas corrigé de sa monomanie d'imprudence et d'audace »43.

\section{Le décor d'une architecture sacrée : La cathédrale Saint-Louis de La Rochelle (1873-1883)}

Il revient au jeune architecte diocésain Aubin Antoine Magloire Brossard (1800-1885) d'avoir achevé à partir de 1849 la cathédrale Saint-Louis de la Rochelle ${ }^{44}$, construite dès 1742 par Jacques Ange Gabriel sur des plans de son père Jacques V : église en forme de croix latine à trois nefs et neuf travées dont un transept peu saillant. Le plan d'Antoine Brossard reprenait, pour l'essentiel, les plans de Gabriel mais dans un souci d'économie, on ajourna la construction des deux tours qui, de part et d'autre de la façade de la cathédrale, devaient en équilibrer la largeur. Des modifications étaient apportées dans la communication des chapelles rayonnantes avec le déambulatoire : arcade unique chez Gabriel, triple baie chez Brossard entre le déambulatoire et les chapelles du Sacré-Cœur et de Notre-Dame-du-Perpétuel-Secours, chapelles symétriques de part et d'autre de la chapelle axiale consacrée à la Vierge. L'ordonnance de cette dernière - où travailla principalement Pascal - fut aussi modifiée : une niche concave remplaça la fenêtre axiale prévue par Gabriel et deux larges niches (ill. 7) furent aménagées dans l'épaisseur des piliers, donnant plus d'ampleur à la chapelle.

\section{7 : Vitrail de la Mort de saint Joseph. Cathédrale Saint-Louis de la Rochelle}

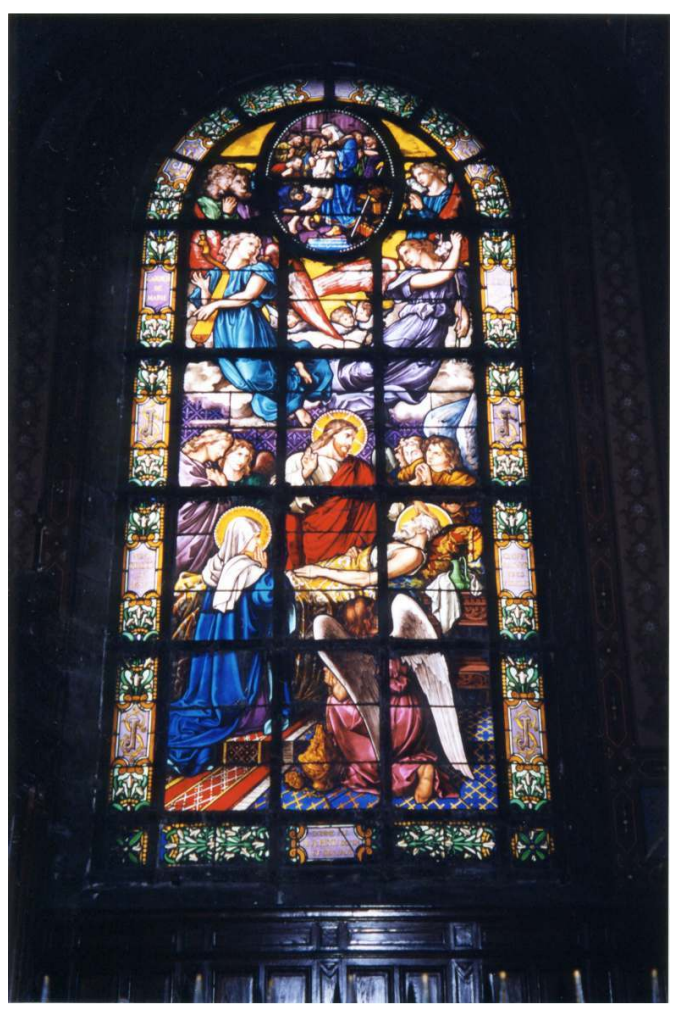

1874. Chapelle Saint-Joseph (4e bas-côté gauche). Cartons : William Bouguereau et Jean-Louis Pascal. Réalisation : ateliers A. Lusson et L. Lefèvre

Cl. auteur. 
18 novembre 1862, la cathédrale était consacrée par l'archevêque de Tours Mgr Guibert. Cependant, tout l'aménagement intérieur de la cathédrale restait à faire. Il le sera entre 1872 et 1892 pour l'essentiel, sous l'épiscopat de Léon Benoît Charles Thomas (1867-1883) et de Pierre Marie Étienne Ardin (1884-1892).

31 L'ornementation des chapelles des bas-côtés et du chœur fut réalisée au hasard des générosités privées et se poursuivit parfois sur plusieurs années ${ }^{45}$. Ainsi le doyen du chapitre, l'abbé Petit, participa à la décoration de la chapelle Saint-Joseph ${ }^{46}$ pour laquelle Bouguereau et Pascal dessinent en 1872 le carton du vitrail (ill.7). Il représente la Mort de Saint Joseph ; ceint d'une bordure décorative aux motifs alternés de cartouches inscrits et de fleurs de lys, il est orné en son cintre d'un médaillon représentant le Joseph de l'Ancien Testament se faisant reconnaître par ses frères. Il fut réalisé par les ateliers parisiens d'Antoine Lusson ${ }^{47}$ et L. Lefèvre. Le chemin de croix, dont Pascal dessina l'encadrement, était dû à la générosité du chanoine Bégaud. Il fut installé dans la nef et le déambulatoire et inauguré le 12 avril 1878. Les cadres en ont été exécutés par le sculpteur Félix Vételet. Il a été peint dans les ateliers parisiens du peintre Cabane, qui était le gendre de Léon Perrault, à partir d'esquisses fournies, semble-t-il, par William Bouguereau. C'est peut-être ce peintre, Léon Jean Basile Perrault (1832-1908), ancien élève de Bouguereau, très proche de lui ${ }^{48}$ et ami de Pascal (qui lui construit une maison ${ }^{49}$, et des immeubles rue du Général Appert ${ }^{50}$, à Paris), qui a réalisé les esquisses ${ }^{51}$, car un chemin de croix extrêmement proche de celui de la Rochelle, et réalisé - selon toute vraisemblance - par Léon Perrault en 1862-1863, est conservé dans les musées de la ville de Poitiers $^{52}$. Léon Perrault avait aussi fourni pour la sacristie de La Rochelle un Saint Jean le Précurseur, médaillé au Salon de 1876, année de son acquisition par l'État.

Antoine Brossard prévoyait des vitraux en grisaille pour huit fenêtres du transept et pour celles de la nef ; ceux-ci furent exécutés en 1873 par la manufacture parisienne d'Antoine Lusson et L. Lefevre qui, entre 1872 et 1874, fournit aussi ceux de trois chapelles dont les chapelles Saint-Joseph et du Sacré-Cœur.

\section{LA CHAPELLE DE LA VIERGE}

La chapelle de la Vierge ${ }^{53}$, qui figurait sur le plan de Gabriel, n'avait pas été exécutée. Elle devait être placée dans l'axe longitudinal de l'église derrière le chœur. La forme était elliptique en plan. C'est l'architecte Antoine Brossard qui l'exécuta d'après les plans de Gabriel. Mgr Thomas, évêque de La Rochelle, projetait de faire peindre l'ensemble de la cathédrale. Seule la chapelle de la Vierge le fut. En 1877, il soumit à la municipalité le projet de décoration de celle-ci ; la municipalité approuva ce projet et vota la moitié de la somme, 20000 francs, l'évêché prenant à sa charge l'autre moitié. La décoration devait être exécutée par le peintre William Bouguereau, qui s'était réservé le droit de faire ce travail à son gré, comme sujets, et comme dates. Le peintre avait subi, entre 1872 et 1877 , la perte de trois de ses cinq enfants, et celle de sa femme ${ }^{54}$. Les toiles qu'il réalise lui sont certainement inspirées par ces drames, notamment sa Pietà, dont la monumentalité rappelle l'œuvre de Michel-Ange. Alors que, jeune pensionnaire de la Villa Médicis, il effectuait un périple en Italie ${ }^{55}$, William Bouguereau avait été enthousiasmé par les peintures murales de la basilique Saint-François à Assise, par les mosaïques de SaintApollinaire et Saint-Vitale de Ravenne; il avait même caressé un temps le rêve de se consacrer à la peinture sacrée. Son éducation première l'y prédisposait : son oncle, curé de Mortagne, lui avait inculqué le sentiment qu'il devait employer les dons reçus du ciel à 
célébrer la gloire de Dieu ${ }^{56}$. Il était donc tout indiqué pour réaliser la décoration de cette chapelle, qu'il n'achèvera qu'en 1883.

La chapelle mariale offre trois divisions principales : les murs percés de fenêtres que séparent des pilastres ioniques couronnés de leur entablement, une voussure élevée, et un dôme (ill. 8).

\section{8 : Chapelle de la Vierge. Cathédrale Saint-Louis de la Rochelle}

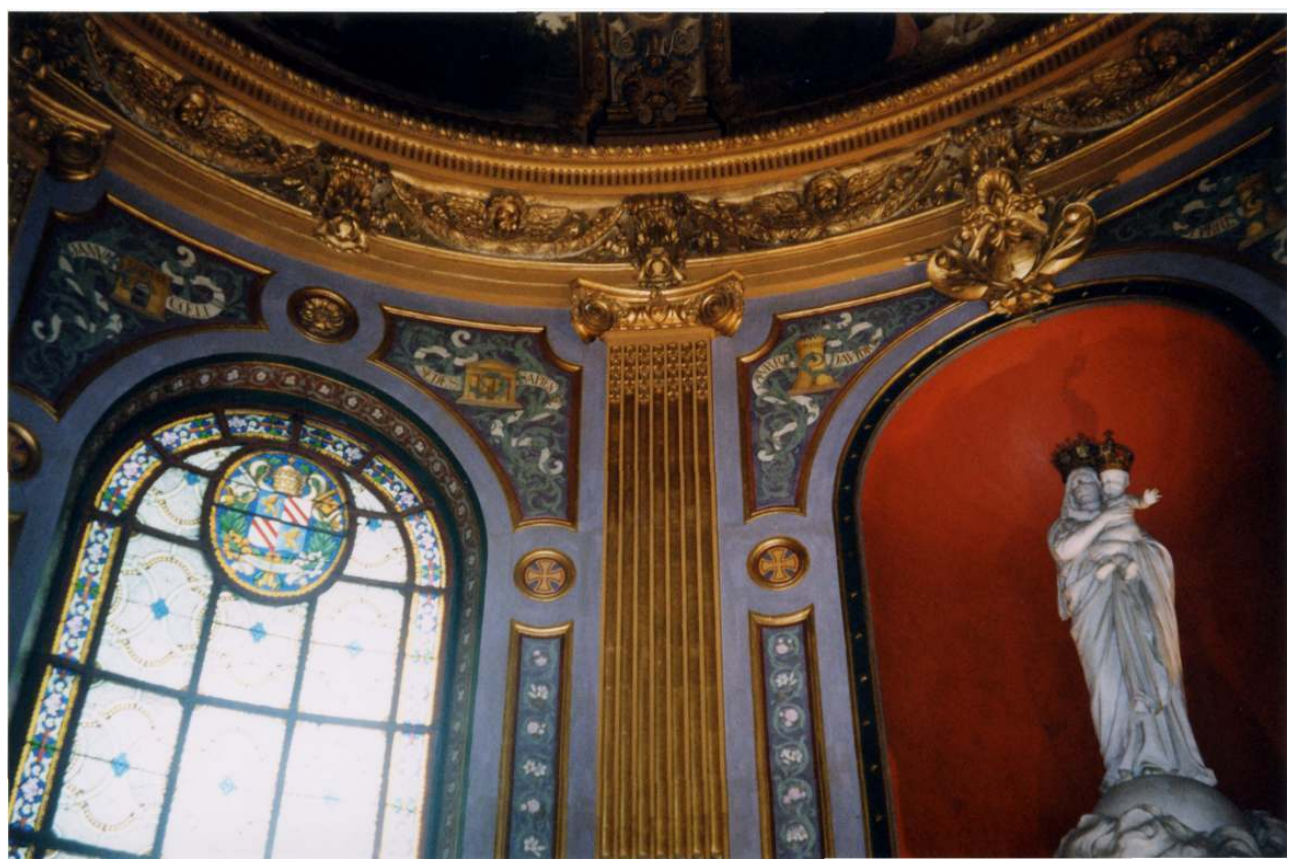

1873-1883. Décoration des murs entre pilastres et fenêtres. Jean-Louis Pascal et Jean-Baptiste Lavastre et Édouard Despléchin. Vitrail : Lusson

Cl. auteur

C'est Jean-Louis Pascal qui réalise les détails des peintures décoratives, à grandeur d'exécution. Elles seront exécutées par Jean-Baptiste Lavastre (1829-1883), le décorateur de l'Opéra de Paris, et le peintre Édouard Despléchin (1802-1871) ${ }^{57}$.

La décoration des murs compris entre pilastres et fenêtres et celle des montants à l'entrée de la chapelle est réalisée à l'aide de lys, et les tympans des arcades sont ornés des emblèmes empruntés aux litanies de la Vierge.

37 La voussure est divisée en compartiments par des parties séparatives feintes, habilement traitées : elles suggèrent des supports prenant appui sur l'ordre ionique au-dessous de la voussure et la raccordant avec le dôme au-dessus, donnant ainsi à l'ensemble un aspect d'unité architecturale riche, varié et très agréable. Chacun de ces supports séparatifs porte un cartouche où est inscrit le monogramme de la Vierge. Des chapelets complètent le cartouche (ill. 9). 
III. 9 : Parties séparatives feintes divisant la voussure de la chapelle de la Vierge. Cathédrale SaintLouis de La Rochelle

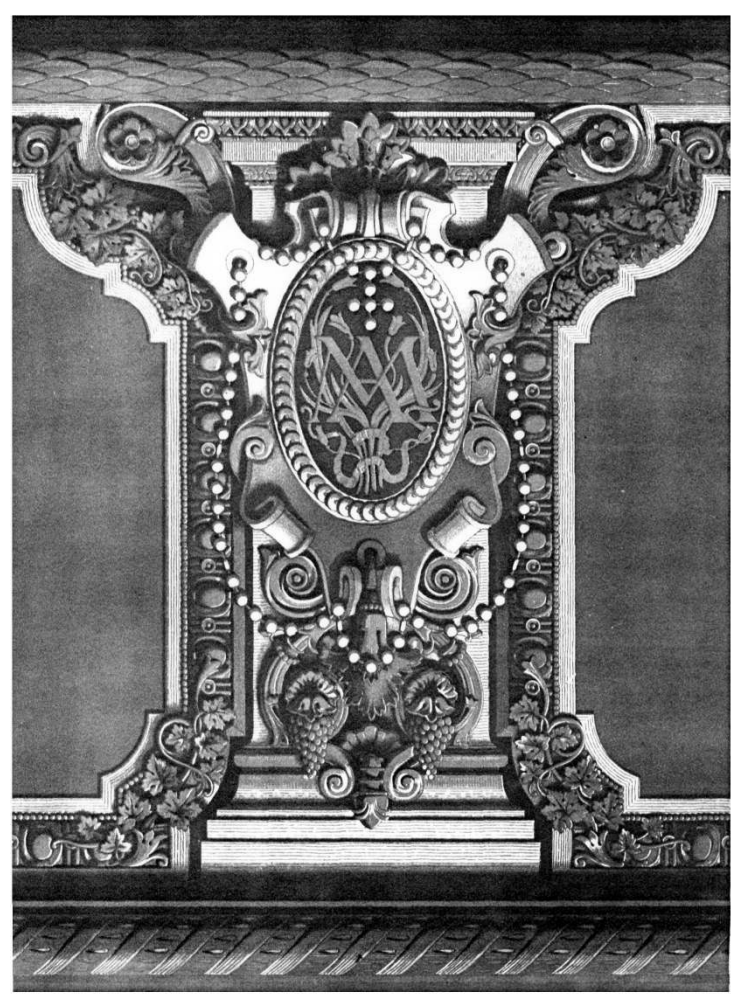

Jean-Louis Pascal architecte et Jean-Baptiste Lavastre et Édouard Despléchin, peintres R.G.A.T.P. 1882, planches 39-40

retombée de la coupole, représentant les principaux événements de la vie de la Vierge. Ce sont six sujets de méditation silencieuse au milieu d'un décor foisonnant de moulures et de dorures : l'Annonciation et la Visitation, la Nativité et la Fuite en Égypte, la Pâmoison de la Vierge et la Pietà. Au dôme le peintre exécute une Assomption ${ }^{59}$. Elle est datée de 1875 et elle contraste, par sa légèreté et sa grande science illusionniste avec l'hiératisme des scènes du soubassement démontrant un « art élégant et un peu froid, au dessin impeccable, très marqué par la Renaissance italienne $»^{60}$. directeur des Cultes, Dumay, écrivit en 1890 à l'évêque de La Rochelle à propos de la décoration de la chapelle du Perpétuel Secours (située à droite de la chapelle de la Vierge et face à celle du Sacré-Coeur), il lui dit :

«J'ajouterai que la cathédrale de La Rochelle possède une chapelle, celle de la Vierge, décorée avec infiniment de goût, et que le peintre devrait s'inspirer de cette décoration pour la chapelle du Perpétuel Secours $\aleph^{61}$.

À la mi-juillet 1869, « cinq blocs énormes de la belle pierre blanche des carrières de Châteauneuf " étaient disposés dans la niche de la chapelle de la Vierge afin que le sculpteur Félix Vételet sculpte sur place une statue sur le modèle de Notre-Dame des Victoires. Pour des raisons inconnues, c'est finalement Gabriel Thomas qui exécutera, sept ans plus tard, cette Vierge en marbre ${ }^{62}$. Le piédestal est l'œuvre de Pascal. À l'entrée de la chapelle, la niche de gauche contient le monument funéraire de Mgr Landriot, pasteur des diocèses de la Rochelle et de Reims due à Gabriel Thomas, belle œuvre de 
marbre qui a reçu une médaille d'or à l'exposition universelle de 1889. C'est Pascal qui en a dessiné le piédestal exécuté en marbre. De l'autre côté de l'entrée, à droite, le tombeau de Mgr Eyssautier, qui présida aux destinées du diocèse de 1906 à sa mort, a été réalisé par le parisien Charles Albert Walhain (1877-1936). Il fut installé dans cette chapelle le 15 octobre 1927.

On avait demandé à William Bougereau de préparer les décorations pour les quatre pendentifs de la coupole de la cathédrale; les peintures décoratives de deux autres chapelles, et des boiseries sculptées avaient été demandées à Pascal. Les projets furent faits, mais ne furent pas réalisés.

Dès son retour de Rome, en 1871, Jean-Louis Pascal avait été nommé inspecteur aux travaux du Louvre et des Tuileries, sous la direction d'Hector Lefuel (1810-1880). Il était spécialement attaché à la reconstruction du pavillon de Flore et de la partie occidentale de la galerie du bord de l'eau' ${ }^{63}$. Pascal, quoiqu'admirant Lefuel, ne s'entendait pas avec lui comme il l'écrivit à Charles Garnier au lendemain de la mort de Lefuel :

"C'était le second chef et le dernier que j'ai eu. Il n'avait rien de ce qui m'a fait et me fera toujours aimer le premier ${ }^{64}$, rien de son obligeance simple, de sa bienveillance sans façon et persistante, que je tâche de prendre pour modèle, reportant sur mes successeurs ce que j'ai trouvé si heureusement au début de ma carrière. Je regrette cependant sincèrement le véritable grand artiste qui vient de mourir, pour cette haute et claire intelligence, cette souplesse d'esprit, cette variété d'aptitudes si indispensables aux architectes, dont je l'avais $v u$ faire preuve en toutes circonstances pendant le temps que je l'ai pratiqué; je le regrette pour son talent, pour l'amitié qu'il aurait pu m'inspirer avec la déférence et le respect. . ${ }^{65}$

Quatre ans plus tard, sa nomination en tant qu'architecte en chef de la Bibliothèque nationale le délivre d'une collaboration douloureuse; il démissionne de l'agence du Louvre en décembre 1875.

\section{NOTES}

1. «Le concours pour la reconstruction de l'Hôtel de Ville de Paris (1872-1873), un échec pour l'architecte Jean-Louis Pascal ", Livraisons d'histoire de l'architecture, $\mathrm{n}^{\circ}$ 19, 2010, p. 111-134.

2. Le peintre Rosa Bonheur (1822-1899) se spécialisa dans les scènes rurales mettant en scène des animaux domestiques (Labourage nivernais, 1848, musée du Louvre). Elle connut un grand succès en France mais aussi en Angleterre et aux États-Unis.

3. Paul Eudel, » Les ateliers de peintres. William Bouguereau », L'Illustration, $1^{\text {er }}$ semestre 1886 , p. 263.

4. Peintre d'histoire qui réalisa de grandes compositions murales à Paris, au Panthéon et à l'Hôtel de Ville, et au Capitole de Toulouse et peignit les plafonds du théâtre de l'Odéon à Paris. Il exécuta des cartons de tapisserie pour la manufacture des Gobelins et illustra notamment les Récits des temps mérovingiens d'Augustin Thierry (1887).

5. Peintre de paysage mais aussi de scènes de genre. Ses vues de la campagne française sont baignées d'une luminosité que l'on retrouve dans les tableaux qu'il réalisa lors de ses voyages en Grèce et en Italie. Élève de Michel Martin Drolling et de Louis Cabat à l'École des beaux-arts, il obtint le second grand prix de Rome en 1849. 
6. Portraitiste virtuose qui connut un grand succès parisien, accéda à tous les honneurs et dirigea l'École française de Rome de 1905 à 1912.

7. Mark Steven Walker, William Bouguereau, Exposition du musée du Petit Palais, Paris, 6 février-6 mai 1984, 266 p., p. 52-53.

8. Sa première épouse, Marie-Nelly Monblachon décède en accouchant de leur cinquième enfant, le 3 avril 1877; très vite, dès la fin de l'année, William Bouguereau veut refaire sa vie avec Elizabeth Gardner, qu'il admire, et qui s'était installée en 1871 au $n^{\circ} 73$ de la rue Notre-Dame-desChamps. La mère du peintre s'y opposant, il ne se remariera qu'en 1896, après le décès de cette dernière. Le $\mathrm{n}^{\circ} 73$ avait été construit par Edmond Le Deschault pour M. Lemercier. Je remercie Mathias Auclair, conservateur de la Bibliothèque-musée de l'Opéra, de m'avoir communiqué l'inventaire des plans conservés dans les archives de l'Opéra, en cours de réalisation, qui m'a permis d'identifier le constructeur du $n^{\circ} 73$ rue Notre-Dame-des-Champs. Edmond Le Deschault aurait-il aidé Pascal pour réaliser l'immeuble sur rue $d u n^{\circ} 75$ de la rue Notre-Dame-desChamps? Aurait-il même contribué à sa réalisation ? La similitude des deux immeubles, les dates très proches de construction (1868 pour l'immeuble Bouguereau et quelques années avant 1871 pour le 73 rue Notre-Dame-des-Champs, puisqu'Elizabeth Gardner s'y installe en 1871) ainsi que le fait qu'Edmond Le Deschault était deuxième inspecteur sur le chantier de l'Opéra de Paris de 1861 à 1874 , alors que Pascal y travaille dès 1861, sont troublants. Edmond Le Deschault n'avait que six ans de plus que Pascal.

9. Marius Vachon, William Bouguereau, op. cit., p. 127.

10. J. M. Larbodière, Reconnaître les façades du Moyen Âge à nos jours à Paris, Paris, 2000, Éd. Massin, $205 \mathrm{p}$.

11. Marius Vachon, William Bouguereau, op. cit., p. 128.

12. Ibid, p. 128.

13. Charles Nuitter, Le Nouvel Opéra Garnier, 1875, Paris, 1999, Claude Tchou, 256 p., p. 65.

14. Mark Steven Walker, William Bouguereau, Exposition du Musée du Petit Palais, op. cit., p. 79.

15. Paul Eudel, » Les ateliers de peintres. William Bouguereau », op. cit., p. 263.

16. François Loyer, Paris XIX siècle, l'immeuble et la rue, Paris, 1987, Fernand Hazan, 478 p., p. 151.

17. Lettre de Jean-Louis Pascal du 31 août 1905 au sculpteur ornemaniste Gustave Germain, qui sera son collaborateur sur de nombreux chantiers, dont la Bibliothèque nationale pendant vingtcinq ans. Elle m'a été communiquée par ses descendants: M. et Mme Patrice Germain que je remercie vivement ainsi que M. Michel-Gasse qui a écrit une biographie du sculpteur (à paraître). Gustave Germain sera choisi et imposé par Charles Girault, l'architecte du Grand et du Petit Palais pour orner ces monuments, fleurons de l'exposition universelle de 1900. Il est l'auteur du groupe Lion et angelot ornant le perron du Palais de la Découverte. Le musée de Sète conserve son œuvre : L'amour s'endort (1886). Son atelier était situé 13 rue Boissonade (36 actuel) à Paris, $\left(14^{\mathrm{e}}\right)$.

18. Je remercie chaleureusement $M$. Yannick le Charbonnier, conservateur régional de l'Inventaire général de Basse-Normandie qui m'a communiqué le dossier concernant le château d'Escures. Le dossier d'Inventaire topographique a été réalisé en 1991-1992 par Bernard Ducouret ; le n de notice est IA00121817. L'édifice n'est pas protégé au titre des Monuments.

Je remercie aussi M. Verly, propriétaire de l'hôtel de la Chenevière (que ce château est devenu), pour m'avoir fait visiter son domaine et m'avoir donné quelques plans signés de Jean-Louis Pascal, plans qu'il avait retrouvés dans le grenier de la propriété lors de son acquisition. Certains plans encadrés de Jean-Louis Pascal décorent le hall et les pièces de réception de l'hôtel.

19. Cecilia Pericoli Ridolfini, Saint-Louis-des-Français. Rome, Bologne, éd. Grafica Editoriale, 1988, $30 \mathrm{p}$.

20. B.E.B.A, Ms 744, Lettres de l'architecte Jean-Louis Pascal à Charles Garnier, op.cit., lettre $\mathrm{n}^{\circ} 24$, Rome, 8 février 1870. 
21. "À la mémoire de Henri Charles Louis comte d'Argy, premier colonel de la Légion romaine, commandeur des ordres de la Légion d'honneur de Pie IX et mort à Rome le XXVI janvier MDCCCLXX. Les officiers de son régiment. Resquiescat in pace ".

22. Le nom lui vient d'un mûrier de Chine planté par Alexandre Lenoir et qui a orné la cour jusqu'au 7 juin 1886, date à laquelle il est tombé et a été remplacé par un arbre plus jeune. L'ancien cloître des Petits-Augustins, transformé par Duban, relie la partie de l'École des beauxarts qui donne sur la rue Bonaparte à celle qui s'étend le long du quai Malaquais. Eugène Müntz, Guide de l'École Nationale des Beaux-Arts, Paris, 1889, Maison Quentin, Compagnie générale d'impression et d'édition, 292 p., p. 217.

23. Jean-Paul Alaux, Académie de France à Rome : ses directeurs, ses pensionnaires, op. cit., p. 113.

24. Louis Hautecoeur, Histoire de l'architecture classique en France, la fin de l'architecture classique (1848-1900), t. VII, Paris, 1957, 575 p., p. 126.

25. Georges Gromort, Histoire abrégée de l'architecture en France au XIXe siècle, Paris, 1924, 142 p., p. 104.

26. Louis Hautecoeur, Histoire de l'architecture classique en France, la fin de l'architecture classique (1848-1900), op. cit., p. 424

27. B.n.F., dp. Mss, N.a.fr. 25093, discours de Henri-Paul Nénot à la mort de Jean-Louis Pascal, au nom de la section d'architecture de l'Académie des beaux-arts, p. 236.

28. Les ornements sont dorés en deux tons différents, or jaune et or vert (couronnes de laurier de l'architrave, grandes palmes appuyées sur le bord extérieur des colonnes, feuilles de chêne accompagnant les fleurs de pavot de la cimaise, guirlande de feuilles de chêne du stylobate).

29. Esquisse terre cuite et plâtre à Paris, musée Carnavalet et musée d'Orsay, grand plâtre à Copenhague, Ny Carlsberg Glyptotek. Édition en bronze par Barbedienne. Antoinette Le Normand-Romain, Mémoire de marbre: la sculpture funéraire en France: 1804-1914, exposition Bibliothèque Historique de la Ville de Paris, 1995, p. 275-278.

30. B.E.B.A., Ms 744, Lettres de l'architecte Jean-Louis Pascal à Charles Garnier, op. cit., lettre $n^{\circ} 79$, Paris, 18 janvier 1876.

31. "Monument élevé dans l'École des Beaux-Arts à la mémoire de Henri Regnault et des élèves morts sur les champs de bataille en 1870 et en 1871 ", Encyclopédie d'architecture, $2^{\mathrm{e}}$ série, t. 7, 1878, p. 39-42.

32. L'expression que les architectes ont cherchée étant, avant tout, celle de l'hommage rendu au sacrifice héroïque et aux mâles vertus, et leur espoir étant que ce monument, dressé en pleine École, entretiendra perpétuellement dans la jeunesse ces sentiments d'abnégation et d'honneur qu'ils ont voulu concentrer dans ce seul mot : patrie ». Ibid., p. 42.

33. Exempté par la loi de tout service de guerre, le jeune lauréat du prix de Rome avait voulu néanmoins s'engager, payant de sa vie, son courage et son patriotisme. Les élus du grand prix bénéficiaient de dispositions du paragraphe 6 de l'article 14 de la loi sur le recrutement de l'armée, qui les exemptaient du service militaire.

34. "Monument élevé dans l'École des beaux-arts à la mémoire de Henri Regnault et des élèves morts sur les champs de bataille en 1870 et en 1871 », Encyclopédie d'architecture, op. cit., p. 42.

35. «Chronique », R.G.A.T.P., $\mathrm{n}^{\circ}$ XXIX, 1872, p. 187 et M. Roger-Ballu, l'Art, 1876, t. III, p. 176-183.

36. Le ministère a porté treize mille francs au budget pour notre petit monument. La souscription en a produit environ douze mille ». B.E.B.A., Ms 744, Lettres de l'architecte Jean-Louis Pascal à Charles Garnier, op. cit., lettre ${ }^{\circ} 45,8$ novembre 1872.

37. Le comité créé à cet effet était composé de : Jean-Baptiste Eugène Guillaume, directeur de l'École des beaux-arts ; Ernest Hébert, ancien directeur de l'École de Rome ; Würtz, de l'Académie des sciences ; Mignet, de l'Académie des sciences morales et politiques ; Guignault, de l'Académie des inscriptions et belles-lettres ; Patin, de l'Académie française ; Isidore Pils, Ambroise Thomas, Ernest Meissonnier et Paul Baudry, de l'Académie des beaux-arts; Bida, officier de la Légion d'honneur ; Coquelin, sociétaire de la Comédie-Française ; Charles Garnier, de l'Institut ; Jules 
Clairin, peintre ; Nicolas Escalier, architecte ; Ernest Barrias sculpteur, Jean-Louis Pascal, Émile Pessard, musicien, Auguste Laguillermie et Jules Jacquet, graveurs en taille-douce, Charles Degeorge, graveur en médailles et sculpteur, Édouard Blanchard, peintre, anciens pensionnaires de l'Académie de France à Rome. « Monument élevé à Henri Regnault et aux élèves de l'École des beaux-arts morts sur les champs de bataille (1870-1871) par MM. E. Coquart et J.-L. Pascal, architectes. ", R.G.A.T.P., $\mathrm{n}^{\circ} \mathrm{XXXV}, 1878$, p. 208.

38. Ce qui était relativement peu, mais les initiateurs de la souscription avaient refusé qu'on prélevât un droit d'entrée à l'exposition générale des œuvres de Regnault au quai Malaquais. «Monument élevé dans l'École des beaux-arts à la mémoire de Henri Regnault et des élèves morts sur les champs de bataille en 1870 et en 1871 », Encyclopédie d'architecture, op. cit., p. 40.

39. B.E.B.A, Ms 744, Lettres de l'architecte Jean-Louis Pascal à Louise Garnier, op. cit., lettre $n^{\circ} 52$, Paris, 9 janvier 1874.

40. Ibid., lettre à Charles Garnier n 68, Paris, 10 février 1875.

41. Auguste Doby, » Inauguration du monument d'Henri Regnault ", L'Illustration, $2^{\mathrm{e}}$ semestre 1876, p. 118.

42. "Monument élevé à Henri Regnault et aux élèves de l'École des beaux-arts morts sur les champs de bataille (1870-1871) par MM. E. Coquart et J.-L. Pascal, architectes. », R.G.A.T.P., $\mathrm{n}^{\circ}$ XXXV, 1878, p. 207-208.

43. B.E.B.A., Ms 744, Lettres de l'architecte Jean-Louis Pascal à Charles Garnier, op. cit., lettre $\mathrm{n}^{\circ} 10$, Florence, 10 juillet 1868 .

44. Dominique Mailles et Jean-Yves Riou, La Cathédrale de la Rochelle. Charente-Maritime, Images du patrimoine, 1985, n 12, Inventaire général des monuments et des richesses artistiques de la France, $64 \mathrm{p}$.

45. Ibid., p. 12. C'est ainsi qu'en 1889 , les verrières de la chapelle des saints anges ( $3^{\mathrm{e}}$ dans le bascôté droit) sont offertes par l'un des vicaires de la cathédrale, l'abbé Bouge (2 $400 \mathrm{fr}$.) et celle des évêques ( $2^{\mathrm{e}}$ dans le bas-côté droit) par l'évêque de La Rochelle, Pierre-Marie Ardin (2 $400 \mathrm{fr}$.). Arch. nat., $\mathrm{F}^{19} 7847$, projet de Lucien Léopold Lobin pour la verrière de la chapelle des saints anges, 20 mars 1889 et lettre de l'architecte diocésain Ernest Massiou au ministre de l'intérieur et des cultes, $1^{\text {er }}$ juin 1889.

46. Quatrième chapelle, bas-côté gauche.

47. La manufacture d'Antoine Lusson père (il décède en 1853) est l'une des quatre ou cinq à avoir été créées en France avant 1840. D'abord installée au Mans, elle ouvrit ensuite une succursale à Paris. Dominique Mailles et Jean-Yves Riou, La Cathédrale de la Rochelle. Charente-Maritime, op. cit., p. 28. Après la mort de Lusson (1876), un projet de Lucien Léopold Lobin (1837-1892) ne reçut pas l'agrément du Comité des édifices religieux pour deux vitraux à grands sujets de l'extrémité des transepts, lequel proposa un autre artiste, Émile Hirsch. Ce dernier fournit en 1880 les deux verrières ainsi que celles du chœur. À l'exception de la chapelle Sainte-Thérèse, pourvue d'un vitrail d'Emmanuel et Charles Tournel en 1932, le vitrage des chapelles de la cathédrale est achevé entre 1881 et 1892 par l'atelier créé à Tours en 1848 par Julien Léopold Lobin (1814-1864) et que dirige alors son fils, Lucien Léopold (1837-1892). Simultanément, la cathédrale est garnie de tableaux et de sculptures dont l'origine est triple. Les uns sont commandés à des artistes de la région : Omer Charlet (1809-1882), Lilia Beltrémieux, Jules-César Robuchon (1840-1922) ; d'autres sont des artistes de renom: Émile Hirsch, Louis Matout (1811-1888), Édouard Debat-Ponsan (1847-1913), Gabriel Thomas (1824-1905) (qui travaillera avec Pascal pour le monument à Garnier). D'autres œuvres proviennent d'églises rochelaises détruites ou désaffectées (SaintBarthélemy).

48. En 1868, il est témoin de l'acte de naissance du troisième enfant de Bouguereau à Paris.

49. Construite en 1876 au ${ }^{\circ} 43$ du boulevard Lannes à Paris.

50. L'un des immeubles subsiste au ${ }^{\circ} 25$ de la rue du général Appert, en 1893. Un an plus tôt, en 1892, Pascal avait réalisé une autre construction pour la famille Perrault au $n^{\circ} 13$ de la rue du 
Général Appert. Cette construction a été détruite et remplacée par un groupe d'immeubles réalisé par les architectes Charles Henri Nicot (1878-1967), Émile Molinié (1877-1964) et l'entrepreneur Félix Audoin, correspondant aux $n^{\text {os }} 46$ de la rue de la Faisanderie $\left(16^{\mathrm{e}}\right)$ et aux $\mathrm{n}^{\text {os }}$ 13 à 21 de la rue du Général Appert $\left(16^{\mathrm{e}}\right)$.

51. Il est vrai que les style des deux artistes est très proche, ceci étant dû à leur passage dans l'atelier de Picot; «Le style gréco-romain apparaît chez Bouguereau et Perrault concomitamment et la confrontation des envois des deux artistes au Salon de 1861 est à cet égard éclairante. [...] Les deux peintres cultivent alors ce même goût d'un antique d'école, révisé par Poussin et des couleurs pâles, fondues et travaillées en glacis selon un dessin très précis qui n'est pas sans rapport avec un ingrisme de la seconde génération, celui d'un Gérôme ou d'un AmauryDuval, par exemple. » Blandine Chavanne, Bruno Gaudichon, Catalogue raisonné des peintures du $\mathrm{XIX}^{e}$ et XX $\mathrm{X}^{e}$ siècles (artistes nés après 1774) dans les collections du Musée de la ville de Poitiers et de la Société des Antiquaires de l'Ouest. Musée de la ville de Poitiers, Société des Antiquaires de l'Ouest, 1988. D'autre part, selon le catalogue, « ni la famille du peintre [Bouguereau] ni les spécialistes de son œuvre ne connaissent le motif de ces compositions et pas davantage de documentation se rapportant à son invention par l'architecte rochelais. "

52. Ibid., tableaux référencés sous les $n^{\text {os }} 514$ à 522. (Le chemin de croix, qui a été acquis par le musée de Poitiers en 1984, est incomplet).

53. César Daly, «La peinture décorative de la chapelle de la Vierge à la cathédrale de la Rochelle », R.G.A.T.P., $\mathrm{n}^{\circ}$ XXXIX, 1882, p. 173-175 et 199-203.

54. Il perd sa quatrième fille, Jeanne-Léontine en 1872, son deuxième fils, Georges, en 1875, et son dernier enfant, William-Maurice, décède peu de temps après sa naissance (avril-juin 1877), naissance qui avait coûté la vie à sa mère, Marie-Nelly Bouguereau.

55. Pendant les vacances de la deuxième année à la Villa Médicis, William Bouguereau fait un voyage avec Paul Baudry, les architectes Lebouteux et Louvet, en Ombrie, Émilie, Toscane ; ils passent quatre semaines à Assise, en pension chez l'habitant. Bouguereau copie les peintures de Giotto et de ses disciples dans la basilique Saint-François. Ils visitent Padoue, Ravenne, mais aussi Venise, Naples et Pompéi.

56. Marius Vachon, William Bouguereau, op. cit., p. 61.

57. Arch. nat., $\mathrm{F}^{19}$ 7846. Devis descriptif des travaux de grosses réparations à exécuter en 1876. Architecte diocésain de La Rochelle, Massiou, 26 septembre 1875.

58. Les panneaux mesurent $4 \mathrm{~m}$. de large et $3 \mathrm{~m}$. de haut.

59. Panneau circulaire de $12 \mathrm{~m}$. de diamètre. L'Assomption, la Visitation, la Nativité, la Fuite en Égypte furent présentées au Salon de 1875 ainsi que le tableau de la Flagellation du Christ de Bouguereau. Émile Bayard, «William Bouguereau », Le Monde Moderne, t. 6, juillet-décembre 1897, p. 841-856. Ce dernier tableau fut offert à la Société des amis de l'art de La Rochelle par Bouguereau; il fut exposé au musée puis mis en dépôt à la cathédrale en 1964. Il y orne le mur gauche de la chapelle des fonts baptismaux, $1^{\text {re }}$ chapelle du bas-côté droit.

60. Michel Chevalier, La France des cathédrales, du IVe au XXe siècle, Rennes, éd. Ouest France, 1997, 511 p., p.

61. Arch. nat., $\mathrm{F}^{19}$ 7847. Lettre du directeur des Cultes, Dumay, à l'évêque de La Rochelle, 14 mai 1890.

62. Dominique Mailles et Jean-Yves Riou, La cathédrale de la Rochelle.Charente-Maritime, op. cit., p. 52-55.

63. Jean-Louis Pascal est nommé inspecteur des travaux de reconstruction de la partie sud des Tuileries, le $1^{\text {er }}$ juillet 1871 : «Monsieur, j'ai l'honneur de vous informer que [...] j'ai nommé M. Pascal, inspecteur des travaux de reconstruction du palais des Tuileries, aux appointements de 250 francs par mois, à partir du $1^{\mathrm{er}}$ du mois courant ». Arch. nat., $\mathrm{AJ}^{64} 213$, 1. Agence du palais des Tuileries et du Louvre, 1871, correspondance officielle, lettre du ministre des travaux publics à Hector Lefuel, 3 juillet 1871. 
64. Entré comme sous-inspecteur aux travaux du nouvel Opéra en 1861, Jean-Louis Pascal en sort en tant qu'inspecteur, pour passer quatre ans à la Villa Médicis. À son retour de Rome, il est nommé inspecteur aux travaux du Louvre et des Tuileries. Son premier "chef » était Charles Garnier.

65. B.E.B.A., Ms 744, Lettres de l'architecte Jean-Louis Pascal à Charles Garnier, op. cit., lettre $n^{\circ} 130,2$ janvier 1881.

\section{RÉSUMÉS}

Les premières réalisations de Jean-Louis Pascal sont l'hôtel particulier et l'atelier qu'il construit, en 1868, pour William Bouguereau au $\mathrm{n}^{\circ} 75$ de la rue Notre-Dame des Champs, à Paris dans le $6^{\mathrm{e}}$ arrondissement, et le château d'Escures à Commes dans le Calvados, réalisé l'année suivante. Le sobre classicisme qui en est la marque se retrouve dans le monument au colonel d'Argy érigé par Pascal, pendant son séjour à la Villa Médicis, en 1870, dans l'église Saint-Louis des Français de Rome. Pour Henri Regnault, son camarade de la Villa Médicis, tombé à ses côtés au combat en 1871, Jean-Louis Pascal livre une de ses plus belles réalisations, pleine de grâce et d'élégance. Dans un angle de la cour du mûrier de l'École des beaux-arts de la rue Bonaparte, un petit temple antique, blanc et or, sert d'écrin au buste fier et énergique du peintre disparu. À partir de 1873, Jean-Louis Pascal participe à la décoration des chapelles de la cathédrale Saint-Louis de la Rochelle et notamment de la chapelle de la Vierge à la coupole peinte par William Bouguereau. En 1875, sa nomination à la Bibliothèque nationale, à la suite d'Henri Labrouste, décédé subitement, le délivre d'une collaboration douloureuse avec Henri Lefuel, l'architecte du palais du Louvre, pour qui il travaillait comme inspecteur depuis son retour de la Villa Médicis

The first realisations of Jean-Louis Pascal are the hotel particulier and the studio built in 1868 for William Bouguereau in the Rue Notre-Dame des Champs (75, in the $6^{\text {th }}$ district of Paris), and, the year after, the castle of Escures at Commes in the Calavados. The sober classicism style we could notice here is also remarkable on the monument for the colonel d'Argy built by Pascal during his stay at the Villa Médicis, in 1870, for the Roman church Saint-Louis des Français. For Henri Regnault, his classmate who fell in battle in 1871 before his eyes, Jean-Louis Pascal offers one of his most elegant and graceful realizations. In a corner of the mulberry tree Courtyard of the École des beaux-arts located rue Bonaparte, a small white and gold temple, appears as a ring box for the painter's bust, proud and energetic. From 1873, Jean-Louis Pascal takes part in the decoration of the chapels of the Saint-Louis cathedral at La Rochelle, especially the Holy Virgin Chapel adorned by William Bouguereau. In 1875, his appointment at the National Library, after the sudden death of Henri Labrouste, lets him free from an uneasy collaboration with Henri Lefuel, the architect of the Louvre, with whom he has been working as an inspector since he came back from the Villa Médicis.

Die ersten von Jean-Louis Pascal ausgeführten Werke sind das 1868 für William Bouguereau errichtete Hôtel particulier mit Atelier in der Rue Notre-Dame-des-Champs Nr. 75 im 6. Arrondissement von Paris sowie das Château d'Escurès in Commes im Departement Calvados aus dem folgenden Jahr. Der diese Bauten kennzeichnende nüchterne Klassizismus findet sich auch in dem von Pascal 1870 während seines Aufenthaltes an der Villa Medici errichteten Denkmal für den Colonel Argy in der Kirche San Luigi dei Francesi in Rom wieder. Eines seiner eindrücklichsten 
Werke schafft Pascal mit dem Denkmal für seinen im preußisch-französischen Krieg an seiner Seite gefallenen Gefährten aus der Villa Medici, Henri Regnault: In einem Winkel der Cour du Mûrier der École des Beaux-Arts in der Rue Bonaparte errichtet Pascal einen in Weiß und Gold gehaltenen grazilen antikisierenden Tempel, der den Schrein für eine ausdrucksstarke Büste des gefallenen Malers bildet. Ab 1873 ist Pascal an der Ausgestaltung der Kapellen der Kathedrale Saint-Louis in La Rochelle beteiligt, insbesondere der von William Bouguereau gestalteten Chapelle de la Vierge. 1875 wird Pascal als Nachfolger des plötzlich verstorbenen Henri Labrouste an die Bibliothèque Nationale berufen, womit seine schwierige Zusammenarbeit mit Henri Lefuel, dem Architekten des Louvre, ein Ende findet, für den er seit seiner Rückkehr von der Villa Medici als Inspektor gearbeitet hat.

\section{INDEX}

Index chronologique : époque contemporaine

\section{AUTEUR}

\section{ANNE RICHARD-BAZIRE}

Anne Richard-Bazire est docteur de l'École pratique des hautes études (section des sciences historiques et philosophiques), diplômée en histoire de l'art contemporain et en archéologie égyptienne. Elle est actuellement chargée du cours de synthèse de l'Histoire de l'architecture occidentale, $\mathrm{XIX}^{\mathrm{e}}-\mathrm{XX}^{\mathrm{e}}$ siècles, à l'École du Louvre. Elle a rédigé différents articles : «L'habitat néo-gothique à Paris au XIX siècle", La demeure médiévale à Paris, Étienne Hamon et Valentine Weiss (dir.), Paris, 2012, Archives Nationales, p. 250-257 ; « La salle Ovale ou l'histoire des dysfonctionnements dans les chantiers parisiens du XIX ${ }^{\mathrm{e}}$ siècle à travers l'exemple de la Bibliothèque nationale ", La Collégialité et les dysfonctionnements dans la décision administrative, JeanMichel Leniaud et François Monnier (dir.), Paris, 2011, École Pratique des Hautes Études, p. 103-112; " Jean-Louis Pascal ou le respect du patrimoine », article mis en ligne sur le site "Archicab", le 4 décembre 2011, http://www.jeanfrancoiscabestan.com/bnfrichelieu.html ; « Le concours pour la reconstruction de l'Hôtel de Ville de Paris (1872-1873), un échec pour l'architecte Jean-Louis Pascal », Livraisons d'histoire de l'architecture, $\mathrm{n}^{\circ} 19,2010$, p.111-134 ; « L'escalier d'honneur de la Bibliothèque nationale de la rue de Richelieu ", Sites et Monuments, $\mathrm{n}^{\circ}$ 207, oct.-nov.-déc. 2009, p. 22-24; « La salle Ovale », Revue de la Bibliothèque nationale de France, $\mathrm{n}^{\circ}$ 30, 2008, 96 p., p. 32-39 ; «La faculté de médecine et de pharmacie de Bordeaux par Jean-Louis Pascal (1876-1888 et 1902-1922) », Livraisons d'histoire de l'architecture, $\mathrm{n}^{\circ} 13,2007, \mathrm{p} .105-120$; « Le ministère de l'agriculture de la rue de Varenne par Emmanuel Brune, l'alliance de la science et de l'art », Livraisons d'histoire de l'architecture, $\mathrm{n}^{\circ}$ 8, 2004, p. 81-94; « Le parvis de Notre-Dame » et « Le square Jean XXIII ", Autour de Notre-Dame, sous la dir. de Alain Erlande-Brandeburg, Jean-Michel Leniaud, François, Christian Michel, Paris, D.A.A.V.P., 2003, 269 p., p. 240-242 et 243-245 ; « Les réalisations de Pascal », et «L'École des beaux-arts » avec Fabienne Doulat, Les bibliothèques parisiennes, architecture et décor, sous la dir. de Myriam Bacha et Christian Hottin, Paris, D.A.A.V.P., 2002, 272 p., p. 115-117 et 132-134 ; « Un siècle de réflexion sur la construction des bibliothèques ", Des palais pour les livres, Labrouste, Sainte-Geneviève et les bibliothèques, sous la dir. de Jean-Michel Leniaud, Paris, éd. Maisonneuve \& Larose, 2002, 189 p., p. 58-69 ; « Jean-Louis Pascal et la création de la salle des périodiques de la Bibliothèque nationale (1883-1936) ", Livraisons d'histoire de l'architecture, $\mathrm{n}^{\circ} 1$, 2001, p. 105-125. Elle a dirigé avec Martin Drouin : La Sélection patrimoniale, Québec, Éditions MultiMondes, coll. Cahiers de l'Institut du patrimoine de l'UQAM, n 13, 2011, 
388 p. Elle a participé en octobre 2001 au colloque international organisé par la Bibliothèque Sainte-Geneviève à Paris : « Henri Labrouste et la Bibliothèque Sainte-Geneviève, Histoire et actualité », en octobre 2009 au colloque : «Victor Laloux, un architecte dans sa ville » organisé à Tours par le Conseil général d'Indre-et-Loire, en juillet 2010 aux journées d'études organisées par le Centre-Ledoux-Université Paris I, la Commission du Vieux Paris et l'Association pour la Sauvegarde et la Mise en valeur du Paris Historique, sur le thème : «BNF Richelieu : un projet en questions »; en octobre 2010, elle a été la coordinatrice scientifique avec Martin Drouin de la Sixième Rencontre internationale des jeunes chercheurs en patrimoine sur le thème de la " Sélection patrimoniale », organisée conjointement par l'Université du Québec à Montréal et l'École des Chartes à Paris, sous la direction de Luc Noppen, Lucie K. Morisset (UQAM) et JeanMichel Leniaud (École des chartes). Elle a participé en janvier 2011 aux Journées annuelles de l'équipe Histoire du droit public et de l'administration, EPHE, sous la direction de François Monnier, directeur de l'UR, Jean-Michel Leniaud et Jean-Miguel Pire sur le thème : « Les dysfonctionnements dans la décision administrative ", en novembre 2011 à la journée d'études de l'équipe Histara de l'EPHE, organisée à l'INHA par Jean-François Belhoste, directeur d'études à l'EPHE, et Isabelle Parizet, maître de conférences à l'EPHE, sur le thème : « Architectures bancaires : entre le Beau et l'Utile », en décembre 2012 au colloque international organisé par l'INHA, Le musée d'Orsay et la Maison des centraliens : « William Le Baron Jenney (1832-1907). De l'École centrale de Paris aux gratte-ciel de Chicago ». Elle a réalisé de mars 2012 à janvier 2013 pour le compte de l'OPPIC les recherches historiques préalables à la restauration des galeries Mansart, Mazarine et de la « chambre de Mazarin » à la Bibliothèque nationale Richelieu sous la direction de l'architecte en chef des monuments historiques A. de Saint-Jouan. Adresse électronique : anne.richardbazire@hotmail.com. 